\title{
ON THE BURNSIDE RING OF A COMPACT GROUP
}

\author{
ROBIN A. GORDON
}

\begin{abstract}
The Burnside ring $A(G)$ of an arbitrary compact group $G$ is defined in analogy to tom Dieck's definition for a compact Lie group. A topology is introduced on the set of conjugacy classes of closed subgroups, and a theorem of Dress on the topology of the prime spectrum of $A(G)$ for profinite $G$ is improved and extended to this general setting.
\end{abstract}

This paper is condensed from part of my doctoral thesis [5], written under the supervision of Professor T. tom Dieck, to whom I am indebted for encouragement and advice.

1. The Burnside ring and its additive structure. Suppose $G$ is a compact group, and define a $G$-manifold as a differentiable manifold $M$ together with a continuous left $G$-action such that, for each $g \in G$, the translation $g \cdot$ : $\boldsymbol{M} \rightarrow \boldsymbol{M}$ is a diffeomorphism. Examples are real $\boldsymbol{G}$-representations and manifolds invariantly imbedded in such.

THEOREM 1.1. If $G$ is a compact group, $M$ a compact effective $G$-manifold, then $G$ is $a$ Lie group and $M$ is a differentiable $G$-manifold.

Proof. Since $M$ is compact and locally connected, it has finitely many connected components, say $M=\amalg_{1}^{n} M_{i}$. For $1 \leqslant i \leqslant n$, set $G_{i}=\{g \in$ $\left.G \mid g\left(M_{i}\right)=M_{i}\right\}$. This is a subgroup of $G$, and, since for any $m_{i} \in M_{i}$ the map $f_{i}: G \rightarrow M$ via $f_{i}(g)=g \cdot m_{i}$ is continuous, while $G_{i}=f_{i}^{-1}\left(M_{i}\right), G_{i}$ is clopen in $G$. Thus $H=\cap_{1}^{n} G_{i}$ is a clopen normal subgroup. Now set $H_{i}=\{h \in$ $\left.H \mid \forall m_{i} \in M_{i}, h \cdot m_{i}=m_{i}\right\}$, so that $H_{i}$ is a normal subgroup of $H$ and $H / H_{i}$ acts effectively on $M_{i}$. Then since $M_{i}$ is connected, Montgomery-Zippin [7, p. 208, Theorem 2] shows $H / H_{i}$ to be a Lie group. Hence $H / \cap{ }_{1}^{n} H_{i}$ is again a Lie group. But since $G$ operates effectively, $\cap{ }_{1}^{n} H_{i}=\{h \in H \mid \forall m \in M, h \cdot m$ $=m\}=\{e\}$, so that $H$ is a Lie group, and with it $G$ since it is open in $G$. That $M$ is then a differentiable $G$-manifold follows from [7, p. 208, Theorem 3].

Now for any $G$-space $X$, let $t(X)=\{g \in G \mid \forall x \in X, g \cdot x=x\}$ be that part of $G$ which acts trivially. Then for a $G$-manifold $M$, the $G$-action is derived from an effective $G / t(M)$-action via the projection $G \rightarrow G / t(M)$. Hence $G / t(M)$ is a Lie group.

Received by the editors May 25, 1976.

AMS (MOS) subject classifications (1970). Primary 22C05, 55E99, 57E10, 57E15; Secondary $54 \mathrm{C} 35$.

() American Mathematical Society 1978 
Corollary 1.2. If $M$ is a $G$-manifold and $H<G$, then the fixed point set $\operatorname{Fix}(H, M)$ is a manifold, and the Euler characteristic $\chi(\operatorname{Fix}(H, M))$ is defined.

Proof. We have $\operatorname{Fix}(H, M)=\operatorname{Fix}(H / H \cap t(M), M)$, the right-hand side being understood in the $G / t(M)$-action. The claim follows since $G / t(M)$ is a Lie group.

As a result of these observations, tom Dieck's definition of the Burnside ring $A(G)$ (see [3, pp. 235-236]) is meaningful for any compact group $G$.

Any homomorphism $f: G \rightarrow G^{\prime}$ gives rise to a ring map $f^{*}: A\left(G^{\prime}\right) \rightarrow A(G)$ defined by lifting a $G^{\prime}$-operation via $f$ to a $G$-operation. If $f$ is surjective, then $f^{*}$ is injective since then subgroups of $G^{\prime}$ correspond one-one to subgroups of $G$ containing $\operatorname{ker}(f)$. Now for any compact group $G$, the system of normal subgroups $H<G$ with $G / H$ a Lie group is directed by inclusion, hence gives rise to a directed inverse system of Lie groups and surjections $\left(G / H ; r_{H, K}: G / H \rightarrow G / K\right.$ for $\left.H<K\right)$. Applying the functor $A($ ) yields a direct system of rings and homomorphisms $\left(A(G / H) ;\left(r_{H, K}\right)^{*}: A(G / K) \rightarrow\right.$ $A(G / H))$. In this sense, we have

TheOREM 1.3. $A(G)=\operatorname{dir} \lim A(G / H)$.

Proof. The projections $p_{H}: G \rightarrow G / H$ yield a coherent system of injective homomorphisms $p_{H}^{*}: A(G / H) \rightarrow A(G)$, hence an injective map

$$
\operatorname{dir} \lim A(G / H) \rightarrow A(G) .
$$

Surjectivity is a direct consequence of Theorem 1.1.

This theorem implies that for profinite $G, A(G)$ is just what Dress calls $\Omega(G)$ (see [4, Appendix B, p. II]).

Now in our framework, the homogeneous spaces which play such a distinguished role in the Lie case are generally not manifolds. In fact, $G / H$ is a $G$-manifold iff $G / \cap_{G} g \mathrm{Hg}^{-1}$ is a Lie group. Let $\phi(G)$ be the set of all conjugacy classes $(H)$ of subgroups of $G$ with $G \overline{/ H \text { a }} G$-manifold and $N_{G} H / H$ finite (the latter to be abbreviated by $\underline{H \ll G}$ ).

LEMMA 1.4. For a surjective continuous homomorphism $f: G \rightarrow G^{\prime}, H^{\prime} \mapsto$ $f^{-1}\left(H^{\prime}\right)$ induces an injective map $\phi(f): \phi\left(G^{\prime}\right) \rightarrow \phi(G)$.

Proof. Let $H=f^{-1}\left(H^{\prime}\right)$. Then since

$$
\begin{aligned}
H & =g H g^{-1} \Rightarrow H^{\prime}=f\left(g H g^{-1}\right) \\
& =f(g) f(H) f(g)^{-1}=f(g) H^{\prime} f(g)^{-1},
\end{aligned}
$$

$N_{G} H \subset f^{-1}\left(N_{G^{\prime}} H^{\prime}\right)$, and since, for $g \in G$,

$$
\begin{aligned}
f(g) H^{\prime} f(g)^{-1} & =H^{\prime} \Rightarrow g H g^{-1} \\
& \subset f^{-1}(f(g)) f^{-1}\left(H^{\prime}\right) f^{-1}\left(f(g)^{-1}\right) \\
& =f^{-1}\left(H^{\prime}\right)=H,
\end{aligned}
$$

$f^{-1}\left(N_{G^{\prime}} H^{\prime}\right) \subset N_{G} H$. Thus $N_{G} H=f^{-1}\left(N_{G^{\prime}} H^{\prime}\right)$ and $H^{\prime} \ll G^{\prime}$ iff $H \ll G$. 
Also, $G / H$ is homeomorphic to $G^{\prime} / H^{\prime}$, so that $G / H$ is a manifold iff $G^{\prime} / H^{\prime}$ is. Thus $\left(H^{\prime}\right) \in \phi\left(G^{\prime}\right)$ iff $\left(f^{-1}\left(H^{\prime}\right)\right) \in \phi(G)$, and $\phi(f)$ is actually a map $\phi\left(G^{\prime}\right) \rightarrow \phi(G)$. Our candidate for a left inverse is $H \mapsto f(H)$, so we must check that this takes $\phi(G)$ to $\phi\left(G^{\prime}\right)$. Since $H<f^{-1}(f(H))$ for any $H<G$, and $\left(f^{-1} f(H)\right) \in \phi(G)$ iff $(f(H)) \in \phi\left(G^{\prime}\right)$, we are through if we show that $(H) \in \phi(G)$ and $H<K$ imply $(K) \in \phi(G)$. If $H<K<G$ and $G / H$ is a manifold, then $t(H)<K, G / K=G / t(H) / K / t(H)$, and $G / t(H)$ is a Lie group, so that $G / K$ is a manifold. If, furthermore, $H \ll G$, then

$$
N_{G} K / K=(G / K)^{K}=(G / t(H) / K / t(H))^{K} \subset(G / t(H) / K / t(H))^{H}
$$

together with Proposition 14 [3, p. 246], show $K \ll G$.

Let $A^{\prime}(G)$ denote the free abelian group on $\phi(G)$. Then there is an additive map $i_{G}: A^{\prime}(G) \rightarrow A(G)$ extending the map $(H) \mapsto[G / H]$.

THEOREM 1.5. $i_{G}$ is a group isomorphism.

Proof. For any surjective homomorphism $f: G \rightarrow G^{\prime}, \phi(f)$ extends to a map $f^{*}: A^{\prime}\left(G^{\prime}\right) \rightarrow A^{\prime}(G)$. Then a proof similar to that of Theorem 1.3 shows that $A^{\prime}(G)=\operatorname{dir} \lim A^{\prime}(G / H)$, the limit being taken over the $G / H$ which are Lie groups. Now the commutativity of

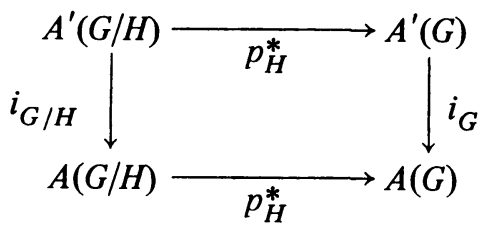

shows that $i_{G}$ is the limit of the maps $i_{G / H}$. These are isomorphisms by tom Dieck [3, p. 239, Theorem 1].

2. Spaces of subgroups. In [6, p. 153, Definition 1.7], Michael introduces a topology on the set of closed nonempty subsets of a topological space $X$, denoted by $2^{X}$, by taking as a subbase all sets of the form $\left\langle U_{1}, \ldots, U_{n}\right\rangle=$ $\left\{A \in 2^{X} \mid A \in \cup_{1}^{n} U_{i}, \forall i, A \cap U_{i} \neq \varnothing\right\}$ with $U_{i}$ open in $X$. In fact, this is a base, and the resulting topology is called the finite topology on $2^{X}$. If $X$ is compact, then $2^{X}$ is compact, and if $f: X \rightarrow Y$ is a continuous closed surjection, then a continuous map $f_{*}: 2^{X} \rightarrow 2^{Y}$ is defined by $f_{*}(A)=f(A)$. If $X$ has the topology defined by a bounded metric $d$, then we can more conveniently topologize $2^{X}$ with the help of the Hausdorff metric $\bar{d}$ defined by

$$
\bar{d}(A, B)=\max \left(\sup _{a \in A} d(a, B), \sup _{b \in B} d(A, b)\right) .
$$

If $X$ is compact metric, then these two topologies coincide.

Now given a compact group $G$, let $S(G)$ denote the set of closed subgroups of $G$. Since any subgroup contains the neutral element, we have $S(G) \subset 2^{G}$. The following two propositions are readily deduced from the definitions.

Proposition 2.1. $S(G)$ is closed in $2^{G}$, hence compact. 
Proposition 2.2. The G-operation on $2^{G}$ given by $A \mapsto g \mathrm{Ag}^{-1}$ is continuous.

The $G$-operation on $2^{G}$ leaves $S(G)$ invariant. We will denote by $c S(G)$ the orbit space $S(G) / G$. It is the space of all conjugacy classes of closed subgroups, and is compact since $S(G)$ is. Note that $\phi(G) \subset c S(G)$.

Now suppose that $I$ is a set directed by an order relation $\leqslant$, and that for each $i \in I$ we have a compact space $X_{i}$, for $i \leqslant j$ a continuous (hence closed) surjection $f_{i, j}: X_{i} \rightarrow X_{j}$. Then we have an inverse system of compact spaces $2^{X_{i}}$ and continuous maps $\left(f_{i, j}\right)_{*}: 2^{X_{i}} \rightarrow 2^{X_{j}}$. The next proposition follows from general properties of limits (see [1, p. 49]).

Proposition 2.3. $2^{\text {inv } \lim X_{i}} \cong \operatorname{inv} \lim 2^{X_{i}}$.

We are interested in the case where the $X_{i}$ are groups $G_{i}$ and the $f_{i, j}$ are continuous surjective homomorphisms. Then by suitably restricting and factorizing the maps $\left(f_{i, j}\right)_{*}$ we obtain inverse systems $\left(S\left(G_{i}\right) ;\left(f_{i, j}\right)_{*}\right)$, $\left(c S\left(G_{i}\right) ;\left(f_{i, j}\right)_{*}\right)$ and $\left(\phi\left(G_{i}\right) ;\left(f_{i, j}\right)_{*}\right)$. Letting $G=\operatorname{inv} \lim G_{i}$, we have

Corollary 2.4. $S(G) \cong \operatorname{inv} \lim S\left(G_{i}\right), c S(G) \cong \operatorname{inv} \lim c S\left(G_{i}\right)$ and $\mathrm{Cl}(\phi(G)) \cong \operatorname{inv} \lim \phi\left(G_{i}\right)$, the closure taken in $c S(G)$.

Note that $\phi(G)$ is generally not closed in $c S(G)$. In fact, in any abelian profinite group there is a sequence of subgroups converging to the zero element each term of which has finite index in the whole group. But for $G$ compact Lie, $\phi(G)$ is closed since any sequence converging to a subgroup then eventually consists of subconjugates of that subgroup.

Proposition 2.5. $c S(G)$ is totally disconnected.

Proof. For $G$ compact Lie, this follows from the fact that $c S(G)$ is a countable metric space. But an arbitrary compact group is the inverse limit of its Lie factor groups. Since the inverse limit of totally disconnected spaces is totally disconnected, Corollary 2.4 finishes the proof.

THEOREM 2.6. If $M$ is a compact $G$-manifold, then the map $\chi_{M}: c S(G) \rightarrow \mathbf{Z}$ defined by $\chi_{M}((H))=\chi\left(M^{H}\right)$ is continuous.

For the proof we need two lemmata.

LEMMA 2.7. Suppose $G$ is endowed with a bi-invariant metric $d$, and $\bar{d}$ is the associated Hausdorff metric on $S(G)$. Then if $X$ is a $G$-space with finitely many orbit types, there is an $\varepsilon>0$ such that $\bar{d}(H, G)<\varepsilon$ implies $X^{G}=X^{H}$.

Proof. For any $K<G$, define $\varepsilon_{(K)}=\bar{d}(K, G)$. Then invariance of the metric $d$ ensures this independent of the representative $K$ of the conjugation class $(K)$. Set $\varepsilon=\min \varepsilon_{(K)}$ where $(K)$ runs through the finite set of isotropy types of $X$ unequal to $(G)$. Since $(H)<(K)$ implies $\bar{d}(H, G) \geqslant \bar{d}(K, G)$, we have that $\bar{d}(H, G)<\varepsilon$ implies $(H) \nless(K)$ for all isotropy types of $X$ except possibly $(G)$. Thus if $X_{(K)}$ denotes the set of points in $X$ with isotropy type $(K)$, then 


$$
X=\cup X_{(K)} \text { and } X^{H}=\cup X_{(K)}{ }^{H}=X_{(G)}{ }^{H}=X^{G} .
$$

LEMMA 2.8. Let $G$ be a compact Lie group, $H<G$, and $X$ a $G$-space of finite orbit type. Then there is an $\varepsilon>0$ such that for all $K<G, \bar{d}(K, H)<\varepsilon \Rightarrow X^{K}$ $\cong X^{H}$.

Proof. We can assume $G$ endowed with a bi-invariant metric. If we denote by $X^{\prime}$ the space $X$ with the restricted $H$-action, then $X^{\prime}$ again has finite orbit type, so applying Lemma 2.7 to $X^{\prime}$ and $H$, we obtain an $\varepsilon_{1}>0$ such that if $K<H$ and $\bar{d}(K, H)<\varepsilon_{1}$, then $X^{H}=X^{K}$. Since, for any $g \in G$, we have $X^{K} \cong X^{g K g^{-1}}$, we must only find an $\varepsilon>0$ with $\bar{d}(K, H)<\varepsilon \Rightarrow \exists g$ $\in G$ with $g \mathrm{Kg}^{-1}<H$ and $\bar{d}\left(g \mathrm{Kg}^{-1}, H\right)<\varepsilon_{1}$. To find such an $\varepsilon$, note that by compactness and continuity of conjugation there is an $\varepsilon_{2}$ with $d(e, g)<\varepsilon_{2}$ $\Rightarrow d\left(g g^{\prime} g^{-1}, g^{\prime}\right)<1 / 2 \cdot \varepsilon_{1}$ for all $g^{\prime}, g \in G$. Applying Bredon [2, Corollary

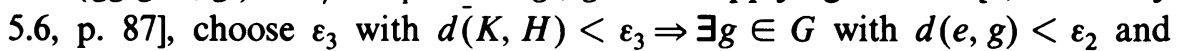
$g \mathrm{Kg}^{-1}<H$. Then

$$
\begin{aligned}
& \bar{d}\left(g K g^{-1}, H\right)=\max _{h \in H} d\left(g K g^{-1}, h\right) \\
& \leqslant \max _{h \in H} \min _{k \in K}\left(d\left(g k g^{-1}, k\right)+d(k, h)\right) \\
& \leqslant \max _{h \in H}\left(\min _{k \in K} d(k, h)+\max _{k \in K} d\left(g k g^{-1}, k\right)\right) \leqslant \bar{d}(K, H)+1 / 2 \cdot \varepsilon_{1} .
\end{aligned}
$$

Thus choosing $\varepsilon=\min \left(\varepsilon_{3}, 1 / 2 \cdot \varepsilon_{1}\right)$ finishes the proof.

ProOF OF THEOREM 2.6. Since manifolds have finite orbit structure, Lemma 2.8 proves our claim for compact Lie $G$. But for general compact $G$, the system of $G / H$ with $H<t(M)$ is cofinal in the system of all Lie factor groups of $G$. $M$ can be considered as a $G / H$-manifold for any such $H$, and $\chi_{M}: c S(G) \rightarrow \mathbf{Z}$ is then continuous as the limit of the continuous maps $\chi_{M}: c S(G / H) \rightarrow \mathbf{Z}$.

Note that the map $\chi_{M}$ in fact depends only upon the class of $M$ in $A(G)$, hence it is meaningful to speak of the map $\chi_{[M]}$ for $[M] \in A(G)$.

3. Prime ideals and Spec $A(G)$. In studying the prime ideals of $A(G)$, tom Dieck introduced the maps $\varphi_{(H)}: A(G) \rightarrow \mathbf{Z}$ defined by $\varphi_{(H)}[M]=\chi_{M}(H)$. Of course, this definition works for arbitrary compact $G$, and the product map $\left(\varphi_{(H)}\right): A(G) \rightarrow \Pi_{(H) \in \phi(G)} \mathbf{Z}$ is still injective. To see this, note that for nonzero $[M] \in A(G)$ there is an $(H) \in \phi(G / t(M))$ with $\varphi_{(H)}[M] \neq 0$. Then if $p: G \rightarrow G / t(M)$ is the projection, $p^{*}(H) \in \phi(G)$ and $\varphi_{(H)}[M]=\varphi_{p^{*}(H)}[M]$.

It is interesting to note that $\phi(G)$ plays a double role in the Lie case. On the one hand, it yields an additive basis for $A(G)$ as in Proposition 1.5, and on the other hand it is the source of all ring homomorphisms $A(G) \rightarrow \mathbf{Z}$ via the construction $\varphi_{(H)}$. In the general compact case, $\phi(G)$ cannot fill the second role as the example after Proposition 2.4 shows: let $H_{i}$ be any sequence of normal subgroups in an abelian profinite group $G$ with limit zero and finite index. The map $\varphi_{(0)}$ carries $\left[G / H_{i}\right]$ to $\left|G / H_{i}\right| \neq 0$. But if $H$ is any subgroup other than 0 , there are certainly infinitely many $H_{i}$ to which $H$ is not 
subconjugate, for which therefore $\varphi_{(H)}\left[G / H_{i}\right]=0$. However, we have

THEOREM 3.1. If $f: A(G) \rightarrow R$ is a ring homomorphism with $R$ an integral domain, then there exists $(H) \in \mathrm{Cl}(\phi(G))$ with $f=\alpha \circ \varphi_{(H)}, \alpha: \mathrm{Z} \rightarrow R$ the map taking 1 to 1 .

Proof. For compact Lie $G$ this is just a simple restatement of [3, p. 244, Proposition 11]. In general, represent $G$ as the inverse limit of its Lie factor groups $G_{i}$, and $A(G)$ as $\operatorname{dir} \lim A\left(G_{i}\right)$ as in Theorem 1.3. Then a ring homomorphism $f: A(G) \rightarrow R$ is the same as a coherent system of homomorphisms $f_{i}: A\left(G_{i}\right) \rightarrow R$. Define $U_{i}=\left\{\left(H_{i}\right) \in \phi\left(G_{i}\right) \mid f_{i}=\alpha \circ \varphi_{\left(H_{i}\right)}\right\}$. Then since each $G_{i}$ is a Lie group, $U_{i} \neq \varnothing$. But if $\left(H_{i}\right)$ is any element of $U_{i}$, then

$$
U_{i}=\bigcap_{[M] \in A\left(G_{i}\right)} \chi_{[M]}^{-1}\left(\varphi_{\left(H_{i}\right)}([M])\right) .
$$

Thus since $\chi_{[M]}: \operatorname{cS}\left(G_{i}\right) \rightarrow \mathbf{Z}$ is continuous, $U_{i}$ is closed in $\operatorname{cS}\left(G_{i}\right)$, hence compact. Now for $i \leqslant j$, the projection $r_{i, j}: G_{i} \rightarrow G_{j}$ yields a continuous map $\left(r_{i, j}\right)_{*}: c S\left(G_{i}\right) \rightarrow c S\left(G_{j}\right)$. The commutativity of the diagrams
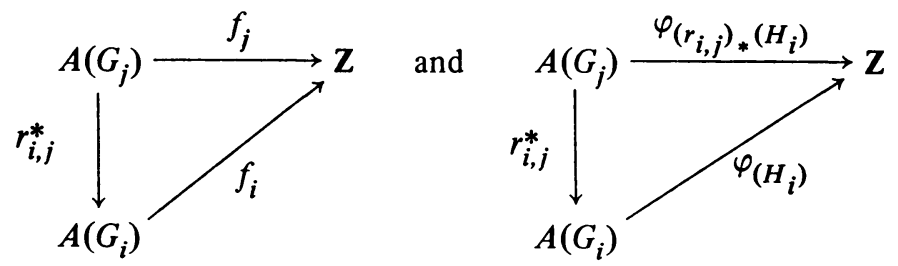

then shows that $\left(r_{i, j}\right)_{*}\left(U_{i}\right) \subset U_{j}$, so that the $U_{i}$ and the restrictions of the $\left(r_{i, j}\right)_{*}$ form an inverse system of compact spaces. The inverse limit of these spaces, say $U$, is then nonempty, and since $U_{i} \subset \phi\left(G_{i}\right), U \subset \operatorname{inv} \lim \phi\left(G_{i}\right)=$ $\mathrm{Cl}(\phi(G))$. Any $(H) \in U$ satisfies the required condition since the projection of $(H)$ to each $G_{i}$ is in $U_{i}$.

Corollary 3.2. Every prime ideal of $A(G)$ is of the form $q((H),(p))=$ $\varphi_{(H)}^{-1}((p))$ for $(H) \in \mathrm{Cl}(\phi(G))$ and $(p) \in \operatorname{Spec} \mathbf{Z}$.

With the information we have gathered, we can now prove a stronger form of a theorem of Dress in the general setting (see [4, Appendix B, Theorem 2.3]).

Theorem 3.3. The map $q: \mathrm{Cl}(\phi G) \times \operatorname{Spec} \mathbf{Z} \rightarrow \operatorname{Spec} A(G)$ is continuous, closed and surjective.

Proof. Surjectivity is just Corollary 3.2. For the other two claims, let $C^{0}$ denote the ring of continuous functions $f: \mathrm{Cl}(\phi(G)) \rightarrow \mathbf{Z}$, latter in the discrete topology. Then there is a map

$$
q_{2}: \operatorname{Cl}(\phi(G)) \times \operatorname{Spec} \mathbf{Z} \rightarrow \operatorname{Spec} C^{0}
$$

defined by $q_{2}((H),(p))=\left\{f \in C^{0} \mid f(H) \in(p)\right\}$. The fact that $\mathrm{Cl}(\phi(G))$ is 
compact and totally disconnected suffices to show $q_{2}$ a homeomorphism. On the other hand, Theorem 2.6 shows that $[M] \mapsto \chi_{[M]}$ defines a map $q_{1}: A(G)$ $\rightarrow C^{0}$, which is a ring homomorphism by the additivity and multiplicativity of the Euler characteristic. Note that since $\mathrm{Cl}(\phi(G))$ is compact, $C^{0}$ is integral over its subring $\mathbf{Z} \cdot 1$. Since $\mathbf{Z} \cdot 1$ is in the image of $q_{1}, q_{1}$ is an integral map, hence induces a closed continuous map

$$
\text { Spec } q_{1}: \operatorname{Spec} C^{0} \rightarrow \operatorname{Spec} A(G) .
$$

But it is easily checked that $q=\operatorname{Spec} q_{1} \circ q_{2}$.

\section{REFERENCES}

1. N. Bourbaki, General topology. I, Addison-Wesley, Reading, Mass., 1966.

2. G. Bredon, Introduction to compact transformation groups, Academic Press, New York, 1972.

3. T. tom Dieck, The Burnside ring of a compact Lie group. I, Math. Ann. 215 (1975), 235-250.

4. A. Dress, Notes on the theory of representations of finite groups. I, Lecture notes, Bielefeld, 1971.

5. R. Gordon, Contributions to the theory of the Burnside ring, Doctoral Thesis, Saarbrücken, 1975.

6. E. Michael, Topologies on spaces of subsets, Trans. Amer. Math. Soc. 71 (1951), 152-182.

7. D. Montgomery and L. Zippin, Topological transformation groups, Interscience, New York, 1955.

Fennstrasse 1, D1000 Beruin 65, Federal Republic of Germany 